\title{
Asbestos related health hazards among power plant workers
}

\author{
Y Lerman, A Finkelstein, Y Levo, M Tupilsky, Mimi Baratz, A Solomon, G Sackstein
}

Studies of asbestos insulation workers have shown a higher than expected death rate due to malignant mesothelioma. ${ }^{1}$ Although asbestos has been widely used in power plants to provide thermal insulation for steampipes and turbines, asbestos related health effects are rarely described among power plant workers $^{2}$ (and J Bonnell et al, XVIII International Conference on Occupational Health, Brighton, 1975). We report two cases of malignant pleural mesothelioma in a clerk and an insulator worker of an electric power plant.

\section{Case report}

A 72 year old man was admitted because of left pleuritic chest pain, non-productive cough, dyspnoea, and progressive weakness. Past and family history were non-contributory. Family history was positive for bone cancer and breast cancer in two siblings. The patient was a retired employee of a power plant where he had worked for 31 years as a clerk until seven years before admission. Before his employment in the power plant he had been a construction worker for five years and a manager in the wood industry for nine years. No history of exposure to asbestos other than in the power plant was obtained.

On examination the patient was malnourished and in mild pulmonary distress. There was no chest wall tenderness or palpable masses. On percussion, the left lung field was dull and breath sounds were appreciably decreased. A chest radiograph showed a left pleural thickening with effusion, the right hemithorax was normal. A pleural tap yielded $800 \mathrm{cc}$ of haemorrhagic exudative fluid. Histological examination of the fluid showed many lymphocytes, histiocytes with few mestothelial cells.

Computed tomography of the chest showed that the left hemithorax was of small volume compared to the right. There was circumferential crenated pleural encasement affecting the left lung and a left basal

\footnotetext{
Israel Defence Forces, Medical Corps, Ichilov Medical Centre, Tel Aviv, Israel

Y Lerman, A Finkelstein, Y Levo, M Tupilsky, M Baratz, A Solomon

Israel Electric Company, Medical Division, Tel Aviv G Sackstein
}

pleural effusion was present. The attentuation numbers of the irregularly thickened pleura were considerably higher than the accompanying free pleural fluid. The suggested diagnosis was of extensive pleural mesothelioma. The presence of a calcified pleural plaque on the right posterior pleural surface indicated exposure to asbestos in the remote past (fig 1).

A pleural biopsy showed a thickened fibrous pleura partially covered by hyperplastic mesothelial cells and fibrin. Within the fibrous stroma were bundles of spindle cells, some with enlarged hyperchromatic nuclei. These findings were interpreted as consistent with cellular fibrous mesothelioma (fig 2). The patient died seven months later from respiratory failure.

\section{Case report 2}

A 54 year old man was admitted because of recurrent pleural effusion. He had been an insulator worker for 31 years and had worked in the same power plant as the first patient. He had no history of exposure to asbestos. On examination, the patient was well nourished and not in pulmonary distress. Expansion of the left chest was decreased. The left lung was dull to percussion and breath sounds were decreased over the same area. A chest radiograph showed a massive left pleural effusion. Pleuroscopy showed the

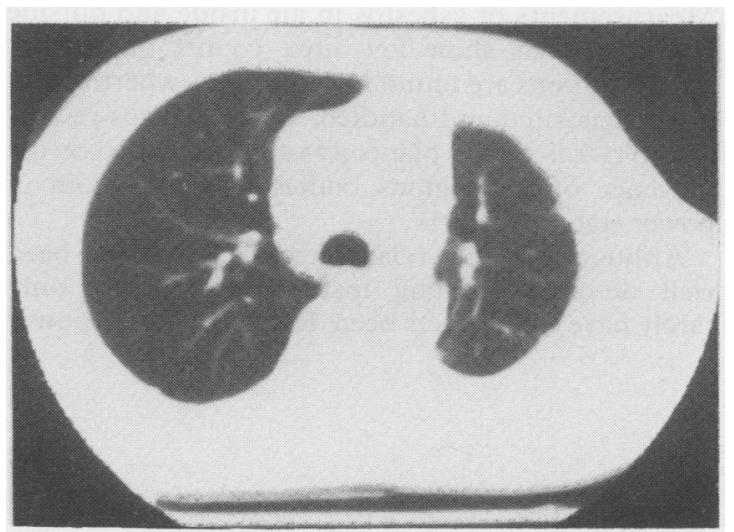

Figure 1 Computed tomography of chest showing encasement of left lung with thick pleural mass accompanied by pleural effusion. Calcified pleural plaque is seen on right posterior surface of pleura. 


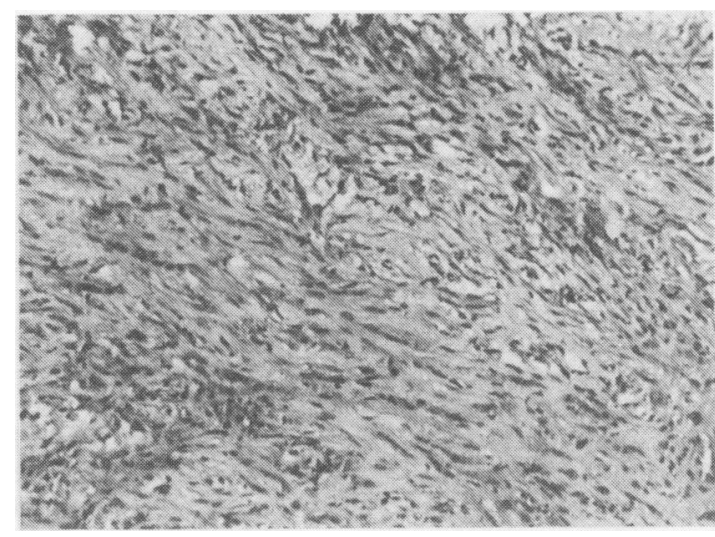

Figure 2 Bundles of spindle cells within fibrous stroma. (Haematoxylin $\&$ eosin $\times 100$ ).

presence of $1500 \mathrm{cc}$ of greenish exudative fluid and a gelatinous mass covering the lower third of the visceral pleura, diaphragm, and pericardium. Biopsy of the pleural mass showed malignant epithelial mesothelioma with many Schauman bodies. The patient received intrapleural treatment with nitrogren-mustard, thiotepa, and yttrium (Itrium) and intravenous treatment with adriamycin. An abdominal tap of ascitic fluid four months later showed malignant mesothelial cells. He died 12 months later from respiratory failure.

\section{Discussion}

Thermal power stations produce electric energy by generators. The generators are complex units consisting of two principal units, the boiler and the turbine. The heat generated by the turbines and steampipes is one of several health hazards in power stations, ${ }^{3}$ and it is essential to provide thermal insulation.

Asbestos has been widely used for this purpose. ${ }^{4}$ Measurements of asbestos in air inside and outside power stations show low fibre counts but higher concentrations are found in storerooms where asbestos is deposited and handled. ${ }^{2}$ The asbestos related health risk in power plants was also confirmed by the presence of ferruginous bodies in the sputum of power station workers. ${ }^{2}$

Although asbestos related health effects have been well described among insulation workers, ${ }^{1}$ only rarely have such effects been described among power station workers ${ }^{2}$ (and J Bonnell, 1975). Bonnell reported on asbestos related disease among 77 insula- $\stackrel{5}{+}$ tion workers of an electric power station in London;을 eight cases of mesothelioma were described. Another $\frac{\overline{\bar{N}}}{\overline{\underline{n}}}$ survey of 55 full time power station workers in $\frac{\bar{s}}{7}$ France showed that the risk of exposure to asbestos $\varrho$ was not confined to workers engaged in laggingis operations: ferruginous bodies and asbestos related $\vec{\circ}$ abnormalities, mainly pleural thickening and cal- cification, were also present among workers in other $\vec{\omega}$ job categories. ${ }^{2}$ In a recent review Cammarano et al $\stackrel{\circ}{\circ}$ reported on site specific mortality among thermo- $\stackrel{\mathbb{D}}{\mathrm{D}}$ electric power plant workers in Italy. ${ }^{5}$ Eighteen? patients with neoplasms were observed whereas only. 8.32 were expected. There were five cases of lung $\dot{i}_{i}^{+}$ cancer (expected 2.83 ) but no cases of mesothelioma.

The nature of exposure of the first patient reported here is a low level long term exposure type. Several authors have suggested that even minimal exposure, $\vec{D}$ such as experienced by maintenance workers ("by-을. stander" exposure) or by neighbourhood or household members may induce malignant meso- $₫$ thelioma. ${ }^{67}$ The risk of asbestosis after this type of. exposure is minimal. ${ }^{1}$ Under such conditions of low level exposure to asbestos cases of mesotheliomas serve as an index of the potential health hazards. The cases reported here emphasise the need for population based studies among power plant workers to $\frac{\mathbb{Q}}{2}$ evaluate the risk accurately.

1 Selikoff IJ, Lee DHK. Asbestos and disease. New York: Academic Press, 1978.

2 Hirsch A, Di Menza L, Carre A. Asbestos risk among full-time workers in an electricity-generating power station. Ann NY

3 Parmeggiani L, ed. Encyclopaedia of occupational health and safety. Vol 2. 3rd ed. Geneva: International Labour Office,

4 Burger H. Asbestos fundamentals. Origin, properties, mining, processing, utilisations. New York: Chemical Publishing Company, 1963.

5 Cammarano G, Crosignani P, Bernino F, et al. Additiona? follow-up of cancer mortality among workers in a thermoelectric power plant. Scand $J$ Work Environ Health 1986;12:631-2.N

6 Epler GR, Gerald MXP, Gansler EA, et al. Asbestos related disease from household exposuure. Respiration 1980;39: N $229-40$.

7 Chen $W$, Mottel NK. Malignant mesotheloma with minimal asbestos exposure. Human Pathol 1978;9:253-8.

Accepted 12 June 1989 